\title{
Le Grand Est : un espace différencié, interface marginale aux portes de l'Europe
}

Great East: a differentiated space, marginal interface at the gates of Europe Der große Osten: Ein differenzierter Raum, Randschnittstelle an den Toren Europas

\section{Sophie de Ruffray}

\section{(2) OpenEdition}

\section{Journals}

Édition électronique

URL : http://journals.openedition.org/rge/619

DOI : $10.4000 /$ rge.619

ISSN : 2108-6478

Éditeur

Association des géographes de l'Est

Édition imprimée

Date de publication : 1 juin 2004

Pagination : 97-106

ISSN : 0035-3213

Référence électronique

Sophie de Ruffray, "Le Grand Est : un espace différencié, interface marginale aux portes de l'Europe », Revue Géographique de l'Est [En ligne], vol. 44 / 3-4 | 2004, mis en ligne le 10 juin 2009, consulté le 08 septembre 2020. URL : http://journals.openedition.org/rge/619 ; DOI : https://doi.org/10.4000/rge.619

Ce document a été généré automatiquement le 8 septembre 2020

Tous droits réservés 


\title{
Le Grand Est : un espace différencié, interface marginale aux portes de l'Europe
}

Great East: a differentiated space, marginal interface at the gates of Europe Der große Osten: Ein differenzierter Raum, Randschnittstelle an den Toren Europas

\author{
Sophie de Ruffray
}

\section{NOTE DE L'ÉDITEUR}

Article reçu le 20 novembre, accepté le 15 décembre 2004

1 Dans la perspective de la construction européenne, le débat sur la création de "grandes régions" au sein du territoire national est récurrent. Les dernières élections européennes au mois de juin 2004 ont vu l'évolution du mode de scrutin, qui, pour la première fois, prévoyait la constitution de listes supra-régionales. Le Grand Est apparait alors comme un espace différencié, dont l'enjeu fondamental est l'influence française dans l'espace européen. L'identité de cette composante a été profondément bouleversée: d'un rempart pour le territoire national, elle est devenue une porte multiple ouverte en direction de plusieurs États, de plusieurs sensibilités avec 940 kilomètres de frontières.

2 L'objectif de cet article est de contribuer à la réflexion sur cet espace en montrant combien cette interface marginale est une zone d'interpénétration de la France et de l'Union Européenne. Malgré les diversités socio-économiques et la divergence des stratégies actuelles de développement, le Grand Est est une entité territoriale complexe, espace intermédiaire entre les dynamiques française et rhénane.

3 Il s'agit ici de mettre en convergence les propriétés intrinsèques du Grand Est et sa situation géographique, avant d'expliciter ensuite les caractéristiques de cette interface 
originale. Cela permettra de souligner dans un troisième temps le rôle fondamental des villes et des réseaux de villes, dans son organisation.

\section{Le grand-est : un espace différencié en situation périphérique}

4 Les concepts de centre, périphérie et marge doivent, avant toute démarche, être explicités en vue de proposer une définition d'un territoire différencié. Les spécificités du Grand Est reposent à la fois sur sa localisation et sur les propriétés intrinsèques qu'elle revêt.

\section{A. Centre, périphérie et marge}

Implicitement, bon nombre d'auteurs se réfèrent aux concepts de centre et de périphérie pour proposer des définitions et des localisations de systèmes régionaux ou locaux. Cette métaphore est aussi utilisée pour décrire l'opposition entre les deux types fondamentaux de lieux dans un système spatial : celui qui le commande et en bénéficie, le centre, et ceux qui le subissent, en position périphérique. Ainsi, pour A. Reynaud (1992), tout territoire, quelle que soit l'échelle spatiale considérée, se subdivise en sousensembles entre lesquels existent des inégalités de développement. Les flux(de personnes, de marchandises, de capitaux, d'informations, de décision...), qui soustendent les relations, doivent être dissymétriques. La plupart des géographes considèrent que le centre est "là où les choses se passent ", c'est-à-dire une certaine masse de population, une capacité notable de production, un niveau de vie élevé, associé aussi à l'idée de pouvoir et d'une capacité d'innovation. A l'opposé, la périphérie se définit négativement par rapport au centre, l'un des principaux handicaps résidant dans l'absence d'autonomie en matière décisionnelle.

C. Grasland (2004) souligne que le système ainsi décrit est autorégulé : le centre reproduit les conditions de sa centralité et réciproquement pour la périphérie. Parce qu'il est fondé sur une logique d'échanges, le système est dynamique. Si certaines périphéries peuvent être délaissées, d'autres peuvent bénéficier de leur situation (position de contact avec l'extérieur du système spatial). C. Rolland-May (2001) définit un espace périphérique comme une frange territoriale ne disposant pas (ou plus) des propriétés systémiques, dont le degré d'organisation est faible - voire inexistant malgré des volontés parfois affirmées (mais souvent non réalisées) de concevoir des structures et une organisation " autonomes » et de mobiliser l'énergie endogène dans un projet d'une ampleur significative. Le caractère-clé est constitué par la fonction distance (éloignement) par rapport à l'espace central. Un espace périphérique est un espace géographique aux limites imprécises et incertaines qui laisse apparaître un territoire-mosaïque à très faible cohésion globale endogène. Si, dans la plupart des cas, le concept « centre-périphérie » explique les phénomènes observés, l'espace de marge nécessite d'avoir recours à d'autres concepts. Espace interstitiel, aux limites d'influence nationale, il est situé loin du centre, à la fois du point de vue de la distance physique mais aussi du point de vue de la distance perçue.

7 Par ailleurs, comme le soulignent des travaux antérieurs (de Ruffray, 2000), un espace de marges possède à la fois des propriétés du centre (capacité d'organisation et d'innovation) et de la périphérie (degré de dépendance notamment en ce qui concerne 
les activités économiques, qui se traduisent par des flux...). Ainsi, C. Rolland-May (2001) définit « un système territorial de marges " comme une frange territoriale possédant des propriétés systémiques, mais dont la fermeture relative réserve l'accès à quelques portes, maintenant le système à un état quasi insulaire, dont certaines caractéristiques fortes traduisent l'enclosure.

Il en résulte qu'un système territorial de marges est une interface, un dispositif destiné à assurer la connexion entre deux systèmes voisins, une zone de contact plus ou moins large, plus ou moins " poreuse ", entre deux systèmes distincts. Une interface est aussi un lieu d'interaction entre deux systèmes, deux organisations; des phénomènes originaux s'y produisent, qui concernent à la fois des échanges ou des modifications entre l'un et l'autre système.

Partant de ces définitions conceptuelles, le Grand Est est non seulement une périphérie de par sa localisation géographique mais aussi une interface marginale, zone de contact et lieu d'interaction entre la France et ses voisins européens comme la Belgique, l'Allemagne et la Suisse.

\section{B. Une délimitation imprécise du « Grand Est »}

Force est de constater que la délimitation du «Grand Est » est doublement imprécise dans l'espace tant du point de vue des "frontières internes» entre ses différents territoires que du point de vue des contours externes.

11 La figure 1 illustre combien la délimitation géographique est plus restreinte que la délimitation politique.

Figure 1 : Une délimitation imprécise du Grand Est.

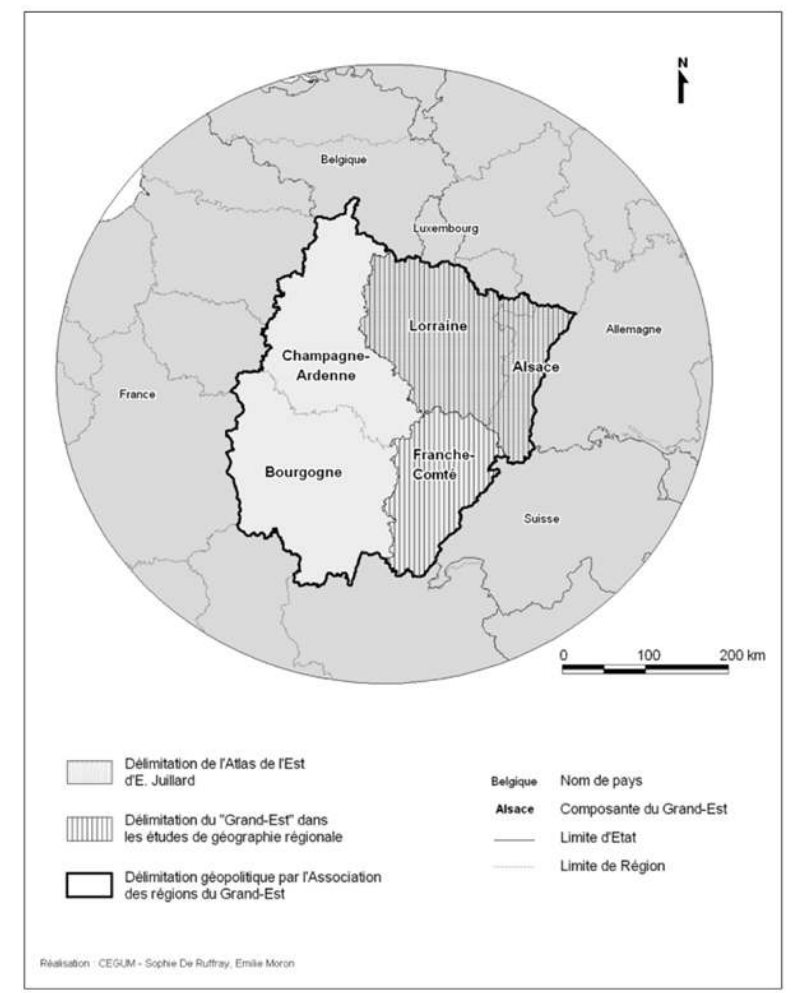


12 À l'échelle des études régionales françaises, la délimitation de l'espace a varié. En 1970, l'édition de «l'Atlas de l'Est » invite déjà à dépasser le cadre régional pour une vision limitée à l'Alsace-Lorraine. En 1977, E. Juillard s'interroge sur les coopérations à trouver entre les régions de la France du Nord-Est.

Plus récemment, G. Wackermann et E. Auphan (1996), dès l'introduction du chapitre sur le Grand Est dans leur ouvrage sur l'aménagement du territoire français, soulignent que «la véritable étude régionale du Nord-Est français est unitaire, les trois composantes Alsace, Franche-Comté et Lorraine - relevant de la même problématique propre à un espace périphérique national qui ne saurait trouver son accomplissement réel que dans sa complémentarité rhénane ».

Depuis quelques années, le Grand Est refait surface comme espace de réflexion porté par le renouveau de la planification territoriale et de l'aménagement du territoire. En 1984, la création de l'Association des Régions du Grand Est, regroupant les cinq Conseils Régionaux d'Alsace, Bourgogne, Champagne-Ardenne, Franche-Comté et Lorraine donne un cadre à la réflexion Grand Est. En 1991, la DATAR retient ce même groupe de régions comme l'un des sept grands chantiers territoriaux de prospective. Dans de nombreuses institutions, organismes, grandes entreprises publics et privés ; le concept de "Grand Est » apparaît pour des raisons d'optimisation organisationnelle avec des implantations territoriales selon des délégations, grandes directions à responsabilité interrégionale. Le concept ne recouvre pas, dans tous les cas, les mêmes entités.

\section{Un espace différencié, fondamentalement tourné vers l'Europe}

La plupart des auteurs s'accordent à identifier le Grand Est comme la plus européenne des interrégions (Nonn, 1996), qui présente l'intérêt d'être à la charnière de l'arc européen et, ainsi, d'être une zone d'ancrage à l'Europe. En effet, sa position géographique dessine une sorte de rectangle dont les deux côtés adjacents forment frontière entre la France et quatre États voisins: la Belgique, le Luxembourg, l'Allemagne et la Suisse. Dans le domaine des relations internationales et particulièrement transfrontalières, cette interrégion, qui dispose du plus grand nombre de voisins immédiats, a une importance stratégique. Ces éléments de positionnement géographique et européen orientent déjà la dynamique propre de développement du Grand Est, qui ne peut qu'inclure une démarche d'échange à partir de ses marges, que ce soit vers l'intérieur ou vers l'extérieur du territoire national. Les évolutions économiques positives observées au cours de la dernière décennie montrent clairement quel atout privilégié pour le développement constitue la position géographique de l'interrégion, au cœur d'un grand espace européen de consommation et de dynamisme.

Le Grand Est se caractérise par une très forte hétérogénéité de développement, avec une concentration sur ses points forts, plutôt en périphérie, et une très forte tendance au développement centrifuge.

17 La répartition de sa population alterne des zones densément peuplées, plutôt jeunes, avec des espaces fortement ruraux. La croissance démographique est tenue et plutôt endogène, à l'exception de l'Alsace. Globalement, le poids relatif de la population du Grand Est est en régression depuis 1982. Cette évolution démographique d'ensemble s'explique par une croissance essentiellement endogène avec un solde naturel en baisse et un solde migratoire négatif. Ainsi, une grande partie du territoire continue à évoluer en creux, notamment les bassins confrontés aux déclins et aux restructurations 
industrielles; seuls certains territoires péri- phériques représentent des zones attractives. Toutefois, cet espace figure parmi les territoires les plus jeunes et contraste beaucoup sur ce plan avec les régions frontalières allemandes.

Les analyses économiques permettent de montrer des situations très contrastées avec des résultats plaçant certains territoires de cet espace dans des positionnements plutôt favorables en ce qui concerne l'implantation des entreprises à capitaux majoritairement étrangers et l'augmentation de l'emploi frontalier, alors que d'autres connaissent des crises profondes.

Enfin, l'absence d'une grande métropole polarisatrice de l'ensemble du territoire fait apparaître un polycentrisme par défaut, éclaté en plusieurs polarités, puisque la plupart des villes majeures se localisent plutôt en périphérie.

À une échelle différente, au regard de l'intégration européenne, le Grand Est représente une entité territoriale réelle mais différenciée, qui distingue plusieurs types de territoires (tableau 1), pas forcément contigus et plus ou moins imbriqués :

- un noyau dur, situé aux confins du territoire national, « cœur » du Grand Est possédant à un degré fort cette aptitude à initier, gérer et optimiser les relations avec l'Europe continentale qui représente la vocation majeure du Grand Est ;

- une bordure pour laquelle cette aptitude est réelle mais dans lesquelles d'autres facteurs se superposent, se combinent ;

- une frange pour laquelle la préoccupation du « Grand Est » n'est plus prédominante et où se dégagent d'autres priorités en matière d'aménagement du territoire, en lien avec les dynamiques de l'agglomération parisienne et de l'agglomération lyonnaise.

Tableau 1 : Les types de territoire du Grand Est.

\begin{tabular}{|c|c|c|c|}
\hline & Situation & Localisation & Caractéristiques \\
\hline $\begin{array}{l}\text { «Cœur » } \\
\text { du Grand } \\
\text { Est }\end{array}$ & $\begin{array}{l}\text { Aux confins du } \\
\text { territoire national, au } \\
\text { contact des voisins } \\
\text { européens }\end{array}$ & $\begin{array}{l}\text { Alsace, Axe mosellan, Arc } \\
\text { frontalier de Longwy à Saint } \\
\text { Louis, Axe Belfort-Montbéliard }\end{array}$ & $\begin{array}{l}\text { - une intégration plus } \\
\text { tournée vers la logique } \\
\text { européenne que nationale } \\
\text { - une capacité } \\
\text { d'organisation } \\
\text { et une dépendance forte } \\
\text { (emplois frontaliers, } \\
\text { investissements } \\
\text { étrangers) } \\
\text { - lieux d'innovation avec } \\
\text { des recompositions } \\
\text { à l'échelle locale et à } \\
\text { l'échelle interrégionale }\end{array}$ \\
\hline
\end{tabular}




\begin{tabular}{|l|l|l|l|}
\hline $\begin{array}{l}\text { Bordure du } \\
\text { Grand Est }\end{array}$ & $\begin{array}{l}\text { Au contact du cœur du } \\
\text { Grand Est }\end{array}$ & $\begin{array}{l}\text { Une partie de la Lorraine, Est de } \\
\text { la Champagne-Ardenne, Nord- } \\
\text { Ouest, de la Franche-Comté, } \\
\text { Ouest de l'Alsace }\end{array}$ & $\begin{array}{l}\text { - influence } \\
\text { transfrontalière plus } \\
\text { nuancée } \\
\text { compte tenu des effets } \\
\text { distances } \\
\text { - zones moins } \\
\text { dynamiques }\end{array}$ \\
\hline $\begin{array}{l}\text { Frange du } \\
\text { Grand Est }\end{array}$ & $\begin{array}{l}\text { Périphérie du Bassin } \\
\text { Parisien et de la } \\
\text { région Rhônalpine }\end{array}$ & Bourgogne et une partie de la \\
Franche-Comté & et démographiquement \\
\hline & & $\begin{array}{l}\text { vis-à-vis des centres } \\
\text { urbains nationaux (Paris, } \\
\text { Lyon) } \\
\text { - flux plus tournés vers } \\
\text { une logique nationale }\end{array}$ \\
\hline
\end{tabular}

\section{Le Grand-Est : une interface aux portes de l'Europe}

21 Compte tenu de la différenciation, le noyau dur, «cœur » du Grand Est, présente les caractéristiques d'une interface dont la vocation est d'initier, de gérer et d'optimiser les flux avec l'Europe continentale. Les infra- structures, l'emploi et les recompositions territoriales à différentes échelles illustrent l'importance et la nature de ces flux.

\section{A. Le rôle des infrastructures dans l'intégration européenne}

La vocation de rempart est devenue une vocation de transit principalement nord-sud, entre des lieux d'origine et de destination qui dépassent largement le territoire national, même si l'arrivée du TGV Est en 2007 réduira la distance avec l'agglomération parisienne, largement après l'ensemble du territoire national.

Les enjeux stratégiques du Grand Est sont devenus indissociables des échanges internationaux et plus particulièrement des échanges transfrontaliers avec la Belgique, le Luxembourg, l'Allemagne et la Suisse. Actuellement, les trafics internationaux de frets routiers, fluviaux et ferroviaires se concentrent de plus en plus sur de grands " corridors » de transport qui associent les trois modes de transport. Ils correspondent également aux zones de plus forte population et connaissent de ce fait, des problèmes de saturation, tant routière que ferroviaire, liés à la coexistence de trafics de transit et de trafics locaux, régionaux, en particulier au droit des agglomérations qui le traversent. En France, ces corridors correspondent à deux itinéraires Nord-Sud :

- entre le Benelux et l'Espagne par Paris et Bordeaux ;

- entre au Nord, le Benelux et l'Allemagne, et au Sud, l'arc méditerranéen (Italie, Espagne) par Lyon et le sillon rhôdanien.

Les régions du Grand Est sont directement concernées par ces flux qui empruntent les couloirs traditionnels que sont le couloir rhénan, le sillon mosellan et le Val de Saône. Ainsi, le sillon mosellan et son prolongement vers Dijon constitue l'axe privilégié de 
transit entre l'Europe du Nord et le sillon rhodanien, tant pour le fret routier que ferroviaire.

\section{B. L'emploi frontalier : dépendance et spécificité}

Comme le montre le tableau 2, le nombre de travailleurs frontaliers ${ }^{1}$ est une des caractéristiques majeures du Grand Est (excepté la Bourgogne). Il représente plus de 60 \% de ce phénomène au niveau national. En effet, pour des zones touchées par le repli des activités sidérurgiques et minières, le travail frontalier constitue une alternative avec des salaires supérieurs à ceux de la France, en moyenne de 15 à $20 \%$ pour ceux qui travaillent en Allemagne et de 25 à $30 \%$ pour ceux qui travaillent au Luxembourg. D'un point de vue spatial, un arc frontalier de Longwy à Saint-Louis (avec une forte croissance endogène et exogène de l'emploi) se singularise à l'échelle du Grand Est.

Tableau 2 : Le poids de l'emploi frontalier dans le Grand Est

\begin{tabular}{|l|l|l|l|}
\hline & Population active en 1999 & Frontaliers en 1999 & Part des frontaliers en 1999 \\
\hline France & 26542481 & 250000 & 0,94 \\
\hline \hline Grand Est & 3652368 & 150692 & 60,28 \\
\hline Alsace & 824353 & 70200 & 28,08 \\
\hline Bourgogne & 710974 & 0 & 0 \\
\hline Champagne-Ardenne & 602963 & 1547 & 0,62 \\
\hline Franche-Comté & 502106 & 13313 & 5,33 \\
\hline Lorraine & 1011972 & 65632 & 26,25 \\
\hline
\end{tabular}

\section{Une recomposition territoriale transfrontalière à des échelles différentes}

Le contexte actuel à l'échelle européenne est celui d'une certaine forme de recomposition des territoires, en raison de l'avancée de l'intégration. Dans ce contexte, le Grand Est français, par sa position géographique est un véritable laboratoire. Les déterminants essentiels en sont d'une part les choix politiques des instances européennes, qui tendent à promouvoir des interrégionalités transnationales et, d'autre part, l'organisation des systèmes économiques qui demandent, pour plus de performances, une polarisation des territoires, associée à un développement des communications.

Le Grand Est est marqué par des initiatives menées à deux échelles différentes avec des agglomérations transfrontalières et des « euro-régions ».

Les premiers exemples concernent les agglomérations transfrontalières entre la France et ses pays voisins, qui sont des ensembles urbains denses et continus d'un point de vue 
morphologique, traversés par une frontière nationale. Si elles se caractérisent par leur extrême diversité, fondée sur leur forte spécificité géographique, le développement des projets de territoire transfrontalier résulte toujours d'une volonté politique forte. Elles témoignent également des dynamiques économiques contemporaines issues d'un certain « effet frontière » avec des différentiels de législation, de coût de main-d'œuvre, de l'immobilier.

Accélérée grâce aux programmes Interreg, la coopération transfrontalière de proximité est plus ou moins complexe selon les accords, les traités, les traditions culturelles. Des problématiques comme les transports, les déchets, la planification font l'objet de coopérations. Ces procédures Interreg ont conféré à l'objectif de construction de territoires locaux transfrontaliers, un statut institutionnel, ce qui permet d'espérer une certaine reconnaissance officielle et plus de durabilité, comme le souligne L. Bertrand (2003).

Comme le montre la figure 2, cinq agglomérations transfrontalières fonctionnelles sont localisées le long des frontières du Grand Est et politiquement portées par les États. Certaines présentent une urbanisation continue et, dans la plupart des cas, une organisation polycentrique avec un centre principal et des centres secondaires.

- l'agglomération transfrontalière trinationale du Pôle Européen de Longwy constitue un projet ancien où les États se sont fortement impliqués financièrement et politiquement depuis 1993. Dédié initialement à la création d'emplois et à la volonté de reconvertir un site en pleine crise après la disparition des activités sidérurgiques, ce projet est devenu multifonctionnel en réponse à une situation locale. La mise en cohérence des documents d'urbanisme des trois pays concernés s'est accompagnée d'une charte d'agglomération et d'un projet de développement, sans oublier des actions concrètes comme un euroguichet social, un centre de formation et un Système d'Information Géographique.

- l'agglomération transfrontalière d'Esch-sur-Alzette/Villerupt/Audun-le-Tiche vise à la recherche de solutions communes aux problèmes d'environnement, la reconversion des friches industrielles, le développement des loisirs et l'amélioration des transports transfrontaliers. Outre une continuité du bâti, son caractère transfrontalier se mesure par l'importance des flux impliqués par le marché du logement (à partir du Luxembourg vers la France en raison de la présence de terrains disponibles à faible coût) et par le marché du travail (à partir de la France vers le Luxembourg). Un projet d'aménagement, avec l'installation d'équipements structurants comme une université, des centres de recherche et des structures d'accueil aux entreprises, est prévu par les luxembourgeois pour soutenir le développement vers le sud du pays.

- l'agglomération transfrontalière Sarrebruck-Moselle Est est portée par l'Association « Zukunft SaarMoselle Avenir » qui a pour objectif le développement et l'approfondissement de la coopération entre les collectivités locales de Moselle et de Sarre. Pour atteindre ces objectifs, l'association développe des initiatives dans les domaines de l'économie, de l'aménagement, du tourisme et de la culture. Avant tout projet, l'agglomération transfrontalière existe de fait, puisque la frontière traverse des zones urbanisées. Avec la réorganisation du marché du travail, Sarrebruck renforce sa fonction de ville centre d'agglomération, alors que l'organisation spatiale était plutôt traditionnellement polycentrique, compte tenu des activités industrielles et minières. A terme, un projet d'Eurodistrict est prévu pour la gestion de cette agglomération transfrontalière.

- l'Eurodistrict de Strasbourg-Ortenau est un projet soutenu par les gouvernements français et allemand pour donner une nouvelle dimension à la coopération transfrontalière en dotant 
l'agglomération Strasbourg-Kehl d'un statut européen. Les groupes de travail mis en place ont permis de définir un périmètre, un groupement local comme organe de décision propre et un certain nombre de projets comme la mise en place des réseaux de transports transfrontaliers et internationaux et le renforcement de la coopération culturelle, de sécurité publique, d'environnement.

- Enfin, l'agglomération Bâle/Saint-Louis/Weil, au point de contact entre trois pays dont un hors de l'Union Européenne, s'est engagée dans un projet global d'agglomération et des actions ponctuelles avec l'objectif d'un rééquilibrage entre ces trois composantes.

Figure 2 : Des recompositions politiques transfrontalières innovantes.

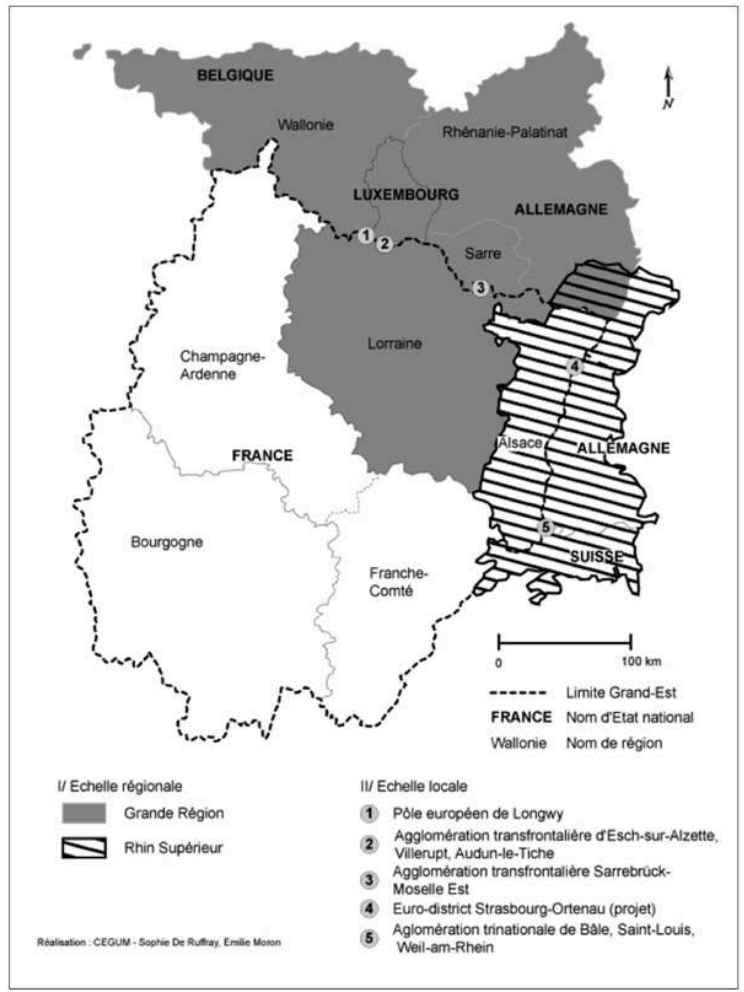

31 Le deuxième exemple concerne des coopérations à des échelles interrégionales avec respectivement la "Grande Région » et l'eurorégion du Rhin Supérieur. A partir de 1995, l'accord confère une plus large liberté aux collectivités territoriales qui peuvent passer des conventions avec leurs homologues européennes pour la réalisation d'équipements d'intérêt commun ou la gestion de services publics.

Héritage de la coopération "Sarre-Lorraine-Luxembourg ", la première regroupe cinq composantes de statuts très différents avec le Grand-Duché du Luxem- bourg, la région Wallone en Belgique, les Länder allemands de Sarre et de Rhénanie-Palatinat et la région Lorraine en France. Coopération interrégionale, la Grande Région regroupe trois formes de coopération différentes: le Sommet pour la coopération politique, l'Assemblée parlementaire consultative pour la coopération consultative et l'EuRegio, pour le niveau communal.

La seconde, l'eurorégion du Rhin supérieur, est le résultat d'un long processus pour mettre en œuvre des collaborations et susciter des structures d'organisation et de fonctionnement transfrontalières dans l'objectif de trouver des solutions aux problèmes posés aux marges actives des États respectifs. Dès 1962, la Communauté 
d'intérêt de la Moyenne Alsace et du Brisgau, à l'initiative des villes de Colmar et de Fribourg, harmonise des projets d'aménagement et mène une action politique en vue de démontrer la relative unité entre les deux sous-régions. La Regio Basilensis, créée en 1963 à Bâle sous la forme d'une association, est chargée de promouvoir le développement économique, social et culturel d'une entité trinationale. Depuis 1995, aucune des régions concernées n'élabore de plan d'aménagement sans y intégrer la facette transfrontalière, largement incitée par l'Europe par l'intermédiaire d'Interreg.

\section{Le rôle majeur des villes dans l'organisation de cette interface marginale}

Dans la perspective de la fonction d'intégration européenne que doit jouer le Grand-Est, le rôle majeur des villes et des réseaux explique l'organisation actuelle de cette interface marginale.

\section{A. Une localisation des grandes villes en périphérie}

À défaut d'avoir un centre, cet espace voit plusieurs polarités s'organiser autour de ses marges, parfois en complémentarité avec des villes extérieures. Il en résulte une distribution spatiale très hétérogène, avec une armature multipolaire dont aucune ville n'a une taille et des fonctions suffisantes pour polariser l'ensemble de l'espace.

Malgré l'importance de ses fonctions tertiaires, Strasbourg ne parvient pas à étendre son rayonnement sur plusieurs régions voisines de l'Est, si ce n'est sur quelques marges lorraines orientales. Bien que partageant certaines composantes du tertiaire supérieur avec Nancy (services aux entreprises, antennes interrégionales d'entreprises...), Strasbourg est handicapée par sa localisation et par certaines conditions médiocres de dessertes. Les fonctions européennes ont des conséquences positives sur le développement économique de la ville et son rayonnement international, mais leurs effets jouent peut-être plus dans le contexte rhénan que dans le contexte Grand Est. L'agglomération de Metz, située à une cinquantaine de kilomètres de la frontière septentrionale de l'Est, se tourne davantage vers Luxembourg et Sarrebruck, que vers le reste de la Lorraine. Le partage de ses fonctions tertiaires avec Nancy lui confère un profil de métropole régionale incomplète. Reims située à la lisière occidentale du Grand Est se trouve autant excentrée que Strasbourg, dont elle n'a pas le rayonnement en tertiaire supérieur privé, ni le rayonnement en fonctions administratives. Dijon, plus méridionale, développe des relations fonctionnelles en direction de Paris et de Lyon. Nancy est la plus grande ville centrale mais son poids est affaibli par le partage des fonctions avec Metz et des liaisons déficientes particulièrement en direction de Mulhouse, Belfort et Besançon. Besançon, quant à elle, n'a pas la taille critique pour jouer un rôle majeur au sein du Grand Est.

37 Ce positionnement périphérique des principales villes de l'Est a également des effets au niveau des organisations fonctionnelles régionales puisque la plupart des armatures urbaines ne sont pas en mesure de structurer entièrement leur région. 


\section{B. Un modèle original d'organisation de villes}

Le Grand Est présente paradoxalement une hiérarchie urbaine transfrontalière de forte connexité (de Ruffray, 2000), sous la forme de modèles d'organisation de «villes en réseau » ou d'agglomérations transfrontalières qui jouent l'interface entre les niveaux hiérarchiques urbains inférieurs et supérieurs. Comme le souligne C. Arbaret-Schulz (1993), une frontière forte (frontière nationale) fournit un appui meilleur qu'une frontière faible (frontière interrégionale) pour impulser une dynamique de coopérations. La première supporte mieux les transgressions et condense davantage de différences (fiscales, salariales, foncières, linguistiques, culturelles).

Sur la figure 3, trois interfaces marginales du Grand Est ont été représentées :

- celle des villes du réseau QuattroPole qui mènent des projets à l'échelle de ces métropoles

régionales ou nationales ;

- celle des agglomérations transfrontalières, interface transnationale à l'échelle des métropoles comme Bâle/Mulhouse ;

- celle des agglomérations transfrontalières ou des réseaux de petites villes transfrontalières.

Figure 3 : Une hiérarchie urbaine transfrontalière de forte connexité

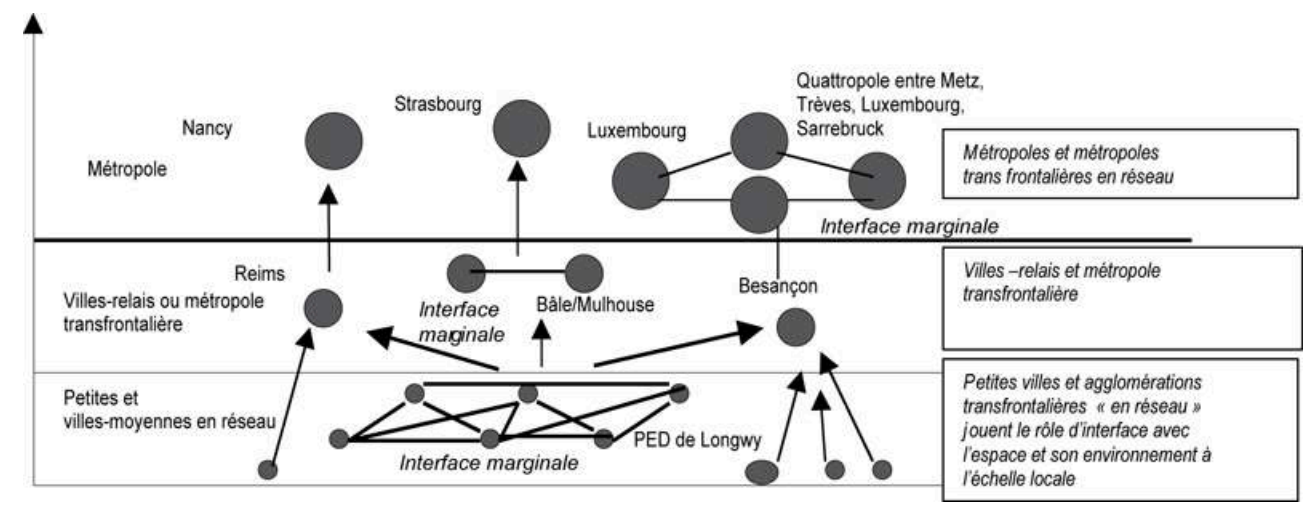

\section{Mise en évidence de l'organisation spatiale du Grand Est}

Les trois types de territoires qui caractérisent le Grand Est, le cœur, la bordure et la frange, se marquent par des implications inégales dans l'intégration européenne, ce qu'il est possible de voir avec la carte (figure 4) dont le commentaire figure ci-dessous. 
Figure 4 : Organisation spatiale du Grand Est.
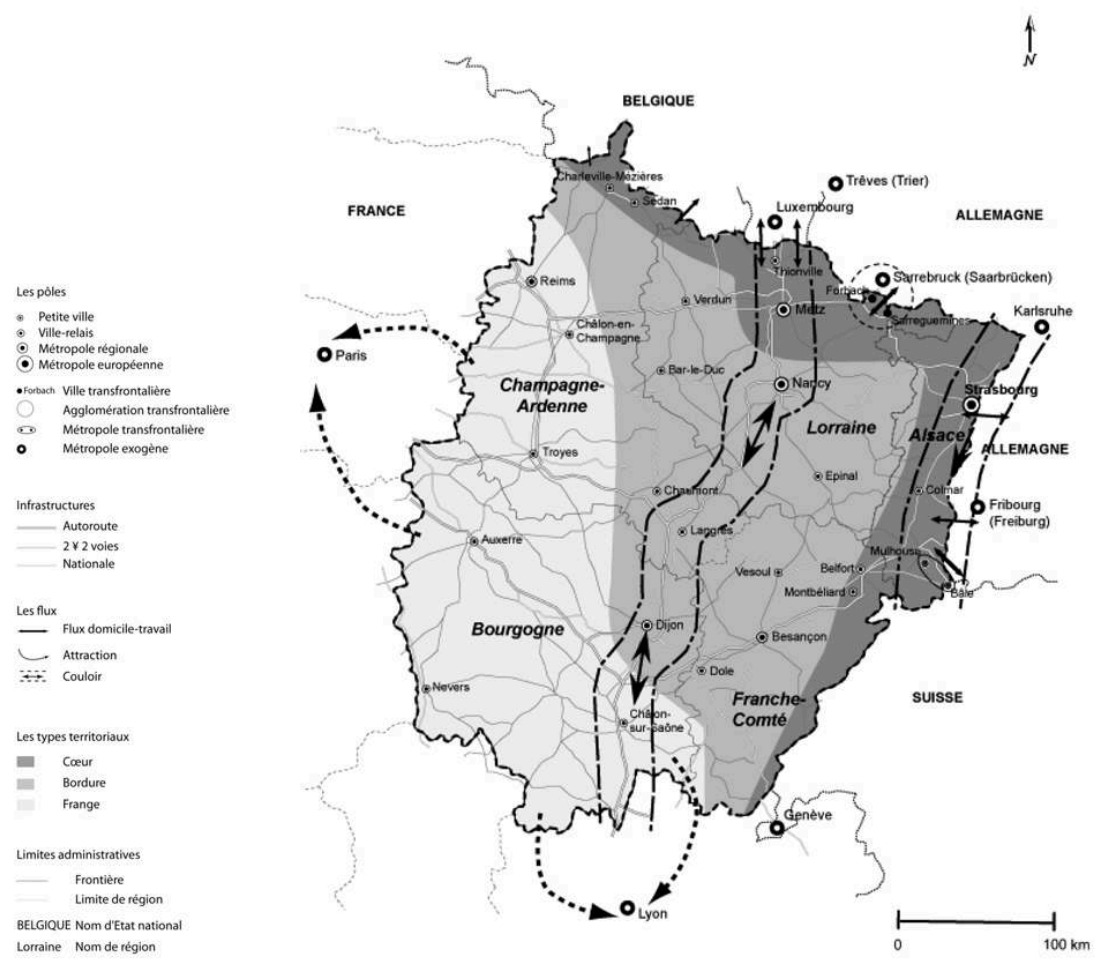

\section{Types de territoires} dont les limites ne correspondent pas aux limites administratives et ont tendance à se rétracter ou à s'étendre en fonction des secteurs. dynamiques de contacts et tous les effets frontières, qu'ils soient économiques, humains et institutionnels. Arc frontalier, ce terrain privilégié de confrontation et d'expérimentation se prolonge naturellement le long des infrastructures qui servent de transit à l'échelle européenne. Les échanges sont maximums depuis l'ouverture des frontières.

Le deuxième type concerne la frange du Grand Est, c'est-à-dire un ensemble plus tourné vers des dynamiques françaises que des dynamiques européennes. Ces caractéristiques sont liées aux aspirations qu'il subit, aux axes qui le traversent et aux pôles qui le structurent avec des gradients différents.

Enfin, le troisième type de territoire constitue la bordure du Grand Est c'est-à-dire un territoire au contact du cœur et de la frange. Ce territoire subit cette double influence transfrontalière et/ou parisienne et/ou rhônalpine. Dans une perspective dynamique, cette bordure est très intéressante car elle constitue pour le décideur le lieu propice pour mener des actions interrégionales à l'échelle du Grand Est. 


\section{Axes et lignes de forces}

Les axes et lignes de forces peuvent être développés en deux sous-ensembles statiques et dynamiques et se matérialisent le plus souvent sous la forme de flux.

En ce qui concerne les axes statiques, les autoroutes et les routes nationales irriguent l'espace d'étude en fonction des contraintes physiques et expliquent une grande partie des dynamiques actuelles, notamment les effets couloirs Nord-Sud. Les limites nationales tendent à s'estomper progressivement avec l'importance des migrations domicile-travail entre la France et ses voisins européens et l'implantation de résidents allemands, suisses et luxembourgeois dans des communes frontalières.

Différents types de flux contribuent à créer et accentuer les gradients à la fois économiques et sociaux du Grand Est :

- des flux transfrontaliers s'exercent dans les régions situées dans l'environnement limitrophe et créent des phénomènes d'aspiration et/ou de diffusion ;

- des flux plus globaux se développent autour des métropoles ou des villes.

Enfin, des effets-couloirs rayonnants qui dynamisent les territoires environnants, situés le long d'axes de communications majeurs, marquent l'espace interrégional.

\section{Pôles d'organisation et stratégies}

Les pôles d'organisation mettent en évidence l'ensemble des niveaux de la hiérarchie urbaine en partitionnant l'espace en trois zones différentes :

- le cœur du Grand Est présente une grande diversité de pôles à tous les niveaux de la hiérarchie urbaine, situés en périphérie ou à proximité, qu'ils soient endogènes, transfrontaliers ou exogènes ;

- la bordure offre des pôles différents qui structurent l'espace en fonction des grands axes ;

- enfin, la frange abrite des pôles fortement dépendants du bassin parisien ou de la région rhônalpine.

Ainsi, le cœur du Grand Est présente les caractéristiques d'une marge territoriale, interface plus ou moins large, plus ou moins poreuse où des phénomènes originaux se produisent. A l'inverse, la frange constitue une périphérie, fortement dépendante de pôles exogènes.

\section{Conclusion}

51 L'enjeu fondamental du Grand Est est celui de l'influence française dans une Europe intégrée, en voie d'élargissement. Cet ensemble territorial diversifié semble pouvoir être désigné non plus comme un ensemble périphérique de l'Hexagone, mais comme un espace de liaison entre les régions françaises et l'Europe médiane.

Des dynamiques économiques ont renforcé depuis de nombreuses années, au sein de l'interrégion, des disparités entre des axes de développement et des espaces intermédiaires, qui leur sont diversement associés. S'opposent ainsi, des zones de marges, territoires plutôt ouverts sur les coopérations et sur les échanges transfrontaliers et des territoires plutôt articulés vers les pôles parisiens et lyonnais. Le Grand Est demeure face à une contradiction fondamentale pour à la fois partager les enjeux européens et atténuer ses oppositions internes. 

contexte européen avec les interconnexions des réseaux de transport avec l'étranger, le renforcement de pôles d'excellence, et la prise en considération de l'extraversion des coopérations et des échanges transfrontaliers comme des opportunités.

\section{BIBLIOGRAPHIE}

ARBARET-SCHULZ C. (1993). - « Sarre-Lorraine-Luxembourg, vers un espace transfrontalier ?». In : Sallez et al., Les villes, lieux d'Europe, DATAR, Editions de l'Aube, p. 129-146.

BERTRAND L. (2003). - « Les territoires transfrontaliers, entre échelle locale et échelle régionale. L'exemple de l'espace Saar-Lor-Lux », Mosella, Tome XXVIII, N 1-2, p. 129-143.

BRUNET R., FERRAS R., THÉRY H. (1992). - Les mots de la géographie, Dictionnaire critique, Paris, Collection dynamique des territoires, GIP Reclus, La Documentation française, $470 \mathrm{p}$.

DATAR, (2000). - « Territoires $2020 »$, Revue d'études et de prospective, n 1, La Documentation française, Paris, $101 \mathrm{p}$.

DATAR (2002). - «Contribution de l'État à de nouveaux enjeux interrégionaux. Le Grand Est », La Documentation Française, 159 p.

GRASLAND C. (2004). - «Centre et périphérie », Hypergéo, http : //hypergeo.free.fr NONN H. (1996). - « Le Grand Est dans la démarche d'aménagement du territoire », Hommes et Terres du Nord, $\mathrm{n}^{\circ}$ 3, p. 158-165

REYNAUD A. (1992). — « Centre et périphéries ». In : BAILLY A., FERRAS R., PUMAIN D., dir. (1992), Encyclopédie de la géographie, Paris, Economica, p. 583-599.

ROLLAND-MAY C. (2000). - «L'évaluation des territoires : concepts, modèle et méthodes », Paris, Hermès, $381 \mathrm{p}$.

ROLLAND-MAY C. (2001). — « Périphéries, bordures, marges territoriales : sous les mots, les concepts ", Regards croisés sur les territoires de marge(s), 2001, Collection RITMA, Strasbourg, Presses Universitaires de Strasbourg, p. 39-60.

RUFFRAY S. DE (2000). — « De la marginalité territoriale à la recomposition territoriale marginale ", Revue Géographique de l'Est, Tome XL, n 4, p. 207-219.

WACKERMANN G. (1996). - « L'aménagement du territoire français », Mobilité spatiale, Paris, Sedes, $467 \mathrm{p}$.

WACKERMANN G. (2003). - « Les frontières dans un monde en mouvement », Carrefours, Paris, Ellipses, $159 \mathrm{p}$.

\section{Sites Internet}

www.espaces-transfrontaliers.org

www.grande-region.lu 
www.quattropole.org

www.regbas.ch

\section{NOTES}

1. Un actif frontalier est, selon la définition communautaire dans le domaine de la coordination des régimes de sécurité sociale des États membres, un travailleur salarié ou non salarié qui exerce son activité professionnelle dans un État membre autre que celui où il réside et dans lequel il retourne au moins une fois par semaine.

\section{RÉSUMÉS}

Dans le contexte de l'intégration européenne, cet article propose une réflexion sur le Grand Est. Aux confins du territoire national, cet espace différencié constitue une interface marginale aux portes de l'Europe. Il a pour objectif de présenter les types de territoires qui le composent et de souligner le rôle des réseaux de villes ou des agglomérations transfrontalières dans la recomposition territoriale. Il insiste sur les enjeux nationaux et européens de cette entité interrégionale.

In the context of European integration, this article reflects on the Great East. Being on the border of its national territory, this differentiated space constitutes a marginal interface with the gates of Europe. The objective of this paper is to present its component territories and to underline the role of the city networks or trans-border agglomerations in territorial recomposition. It underlines the national and European possibilities of this interregional entity.

Im Rahmen der europäischen Integration schlägt dieser Artikel eine Überlegung über den Großen Osten vor. Am äußersten Rand des Staatsgebietes bildet dieser differenzierte Raum eine Randschnittstelle an den Toren Europas. Er hat zum Ziel, die Typen von Territorien vorzustellen, die es zusammensetzen, und die Rolle der Stadtnetze oder der grenzüberschreitenden Agglomerationen in der Neukomposition der Territorien $\mathrm{zu}$ unterstreichen. Schließlich unterstreicht er die nationalen und europäischen Bezüge dieses interregionalen Gebildes.

\section{INDEX}

Schlüsselwörter : grenzüberschreitende Agglomeration, Großer Osten, Peripherie, Schnittstelle, Territoriale Neuordnung

Mots-clés : agglomération transfrontalière, Grand Est, interface, périphérie, recomposition territoriale

Keywords : fringe, Great East, interface, territory recomposition, transborder urban area 
AUTEUR

SOPHIE DE RUFFRAY

CEGUM, Centre d'Etudes Géographiques de l'Université de Metz, Université de Metz, île du Saulcy, 57045 Metz Cedex 1, s.de.ruffray@sha.univ-metz.fr 